\title{
Optimal Ratio of Lamé Moduli with Application to Motion of Jupiter Storms
}

\author{
Ramūnas Girdziušas and Jorma Laaksonen \\ Helsinki University of Technology, Laboratory of Computer and Information Science, \\ P.O. Box 5400, FI-02015 HUT, Espoo, Finland, \\ Ramunas.Girdziusas@hut.fi, Jorma.Laaksonen@hut.fi
}

\begin{abstract}
Fluid dynamics models the distribution of sources, sinks and vortices in imaged motion. A variety of different flow types can be obtained by specifying a key quantity known as the ratio of the Lamé moduli $\lambda / \mu$. Special cases include the weakly elliptic flow $\lambda / \mu \rightarrow-2$, often utilized in the Monge-Ampère transport, the Laplacian diffusion model $\lambda / \mu=-1$, and the hyper-elliptic flow $\lambda / \mu \rightarrow \infty$ of the Stokesian dynamics. Bayesian Gaussian process generalization of the fluid displacement estimation indicates that in the absence of the specific knowledge about the ratio of the Lamé moduli, it is better to temporally balance between the rotational and divergent motion. At each time instant the Lamé moduli should minimize the difference between the fluid displacement increment and the negative gradient of the image mismatch measure while keeping the flow as incompressible as possible. An experiment presented in this paper with the interpolation of the photographed motion of Jupiter storms supports the result.
\end{abstract}

\section{Introduction}

Fluid-based estimation of motion in images covers two major application areas: medical image registration [2] and optical flow estimation [3]. In this work we focus on the latter type of applications, in particular, we attempt to interpolate the cloud movement in Jupiter by means of fluid motion estimation.

The fluid dynamics framework 2 extends the previous works based on linear elasticity aiming at the estimation of large nonlinear deformations. A common problem here is that the displacement estimation qualitatively depends on the ratio of the Lamé moduli $\lambda / \mu$, usually defined in an ad hoc way. Various spatial models of the Lamé moduli have already been considered [7]. However, the possibility of modifying these parameters adaptively in time has not gained considerable attention so far.

Research on fluid dynamics applications often avoids the two key concepts which are in modern analysis known as the ellipticity and point-wise stability of the Lamé functional. A safe constraint $\mu>0$ and $\lambda>0$ is often employed, which, however, misses weakly elliptic modes of the fluid flow. On the other hand, ellipticity constraint $\mu>0$ and $\lambda+2 \mu>0$ is not enough to ensure that a numerical algorithm will inherit essential properties of the continuous problem. 
This work utilizes the ellipticity and point-wise stability concepts and indicates how two resulting sets of constraints on the Lamé moduli affect the models built to estimate a fluid flow.

After simplifying a fluid dynamics model, we view the estimation of displacement increment at each time instant as obtaining the average of a certain Gaussian process (GP). As a result, the Lamé moduli can be interpreted as hyperparameters that maximize the Bayesian evidence criterion [6]. This criterion has already been used to select the weighting factors of some deformable surface energies in order to correct three-dimensional SPECT images [4, but its implications to time-dependent fluid flow are very much unexplored.

The paper is organized into four remaining sections. A concise summary of fluid dynamics models and key difficulties in identifying fluid motion are presented in Section 2 Section 3 develops the Bayesian evidence-based optimality criterion and a realistic algorithm which could be used in temporal adaptation of the Lamé moduli. Numerical results are presented in Section 4. Here we first depict a diversity of deformations caused by a variety of different Lamé moduli and later present the result of an experiment with the interpolation of cloud movement in Jupiter atmosphere. Finally, conclusions are drawn in Section 5.

\section{Fluid Dynamics Models of Image Motion}

Let us assume that the template and target images are smooth real-valued functions $\varrho_{0}(\mathbf{x}) \in \mathbb{H}^{1}(\Omega)$ and $\varrho_{T}(\mathbf{x}) \in \mathbb{H}^{1}(\Omega)$, respectively, defined on the spatial domain $\mathbf{x} \in \Omega=[0,1] \times[0,1]$, i.e. they belong to the space of functions which are square-integrable up to their first order spatial derivatives. The goal will be to find the displacement vector field $\mathbf{u}_{T}(\mathbf{x}) \in\left[\mathbb{H}_{\partial \Omega}^{1}(\Omega)\right]^{2}$, defined over the spatial domain $\mathbf{x} \in \Omega$, which maps the target image $\varrho_{T}(\mathbf{x})$ onto the template image $\varrho_{0}(\mathbf{x})$ via $\varrho_{T}(\mathbf{x})=\varrho_{0}\left(\mathbf{x}-\mathbf{u}_{T}(\mathbf{x})\right)$ and also satisfies some additional requirements.

Consider first the functionals $\left[\mathbb{H}_{\partial \Omega}^{1}(\Omega)\right]^{2} \rightarrow \mathbb{R}$, defined by

$$
\begin{aligned}
\mathcal{J}[\mathbf{u}] & =\left\|\varrho_{0}(\mathbf{x}-\mathbf{u})-\varrho_{T}(\mathbf{x})\right\|^{2}, \\
\mathcal{R}_{\lambda, \mu}[\mathbf{u}] & =\left\|\nabla^{\perp} \cdot \mathbf{u}_{t}\right\|^{2}+(\lambda / \mu+2)\left\|\nabla \cdot \mathbf{u}_{t}\right\|^{2},
\end{aligned}
$$

where $\|\cdot\|^{2}$ denotes the squared Euclidean norm integrated over the domain $\Omega$ and $\nabla^{\perp} \cdot \mathbf{u}=\partial_{x_{1}} u^{(2)}-\partial_{x_{2}} u^{(1)}$ is a two-dimensional curl operator. We next define the fluid motion model which: (i) sets initial displacement values to $\mathbf{u}_{0}=\mathbf{0}$ and (ii) updates them by adding small displacement increments $\mathbf{u}_{t+1}=\mathbf{u}_{t}+\Delta \mathbf{u}_{t}$ according to

$$
\Delta \mathbf{u}_{t} \approx \arg \inf _{\delta \mathbf{u}}\left(\delta \mathcal{J}\left[\mathbf{u}_{t}\right]+\delta^{2} \mathcal{R}_{\lambda, \mu}\left[\mathbf{u}_{t}\right]\right)
$$

Here the functionals $\delta \mathcal{J}\left[\mathbf{u}_{t}\right]$ denote the first and second variation of Eqs. (11) and (2) respectively, where the latter one is also weighted with $c_{t}>0$. The first functional is linear in $\delta \mathbf{u}$ whereas $\delta^{2} \mathcal{R}\left[\mathbf{u}_{t}\right]$ is quadratic in $\delta \mathbf{u}$. The term $\delta^{2} \mathcal{J}\left[\mathbf{u}_{t}\right]$ encompasses the second-order effects of the optical flow and can be neglected. 
The existence and uniqueness of the stationary state $\mathbf{u}_{t \rightarrow T}$ then entirely depends on the Lamé functional $\mathcal{R}_{\lambda, \mu}\left[\mathbf{u}_{t}\right]$.

Eq. (3) models a fluid motion without inertial terms. Here we further simplified it by neglecting the multiplying matrix $\mathbf{I}-\nabla \mathbf{u}_{t}$ which would normally appear in Eq. (3) due to the Eulerian reference frame [2]. Our experience indicates that in the applications considered below this multiplying factor is not important.

It is the absence of the linear-in- $\delta \mathbf{u}$ or $\nabla \delta \mathbf{u}$ term $\delta \mathcal{R}$ that makes Eq. (3) crucially different from variational regularization problem solving $[5]$. This is the key defining feature of fluid motion: whenever a fluid particle gets pushed, there will be no re-action to force it back. The smoothness here is only due to the particle's interaction with its neighbors via $\delta^{2} \mathcal{R}\left[\mathbf{u}_{t}\right] \neq 0$.

Eq. (3) possesses unique minima if the Lamé moduli satisfy either one of the following requirements:

$$
\begin{aligned}
& \text { Ellipticity: } \mu>0, \quad \lambda+2 \mu>0, \\
& \text { Point-wise stability: } \mu>\mu_{0}, \quad \lambda+\frac{3}{2} \mu>\lambda_{0},
\end{aligned}
$$

for some positive constants $\mu_{0}$ and $\lambda_{0}$. A computational analysis of these two sets of constraints is presented in Section 3.2. We emphasize that Eqs. (4) and (5) are different in the three-dimensional case 5 .

Numerous fluid motion estimation algorithms essentially differ in the application-dependent image error measure $\mathcal{J}$ and in the variants of the Lamé functional. The case $\lambda / \mu=-1$ ca be implemented by performing the steepest descent on the image mismatch with Gaussian smoothing of the displacement increments. Compressible Stokes models and Monge-Ampère transport allow a more diverse range of displacements by considering $\lambda / \mu \rightarrow \infty$ and $\lambda / \mu=-2$ cases, respectively.

We will apply Eq. (3) with the boundary conditions $\left.\delta \mathbf{u}\right|_{\partial \Omega}=\mathbf{0}$. Another constraint will be imposed on the maximum Euclidean norm of the displacement increment $\max \|\delta \mathbf{u}\|_{2}=\kappa$, which removes the need to consider time-dependent regularization weight $c \equiv c(\kappa)$. In general, the absolute values of the Lamé moduli depend on the constant $\kappa$. However, we believe that the choice of $\kappa$ does not produce qualitatively different types of flow, it rather affects its stability. In what follows we replace the ratio $\frac{\lambda}{\mu}$ by a single parameter $\lambda$.

Finding the optimal ratio of the Lamé moduli is challenging for many reasons. If Eq. (3) includes the extra term $\delta \mathcal{R}$, e.g. when it becomes linear elasticity equation, this ratio can be chosen according to the material properties of the imaged objects [7]. A more general methodology considers the joint probability distribution of both: the displacements and the Lamé moduli. A research example in this direction is the application of the Kalman filter to estimation of material properties of the myocardial tissues 9 .

Nice compressibility patterns hardly exist or it is impossible to track them in the imaged fluid motion. Moreover, the functional $\mathcal{J}$ is always a very crude approximation to how image intensity values change along the motion path. These discrepancies would require adequate changes in the Lamé moduli. The 
weaknesses in choosing the Lamé moduli based on the physics of the problem alone can be especially well seen from Bayesian viewpoint.

\section{Image Motion with Adaptive Lamé Constants}

\subsection{Gaussian Process of Fluid Displacement Change}

In order to understand the ellipticity implications and to reduce Eq. (3) to a computationally feasible level, let us approximate the Lamé functional with central finite differences. Eq. (3) then becomes

$$
\mathbf{P}_{\lambda_{t}} \Delta \mathbf{u}_{t}^{*}=\mathbf{f}_{t}+\mathbf{b}_{\lambda_{t}}
$$

where the vector $\Delta \mathbf{u}_{t}^{*}$ consists of the unknown displacement increments at the internal nodes $\mathbf{x}_{1: n}=\left\{\mathbf{x}_{i} \mid i=1,2, \ldots, n=(w-2)^{2}, \mathbf{x}_{i} \in \mathbb{R}^{2}\right\}$ of the discrete domain $\Omega$ with $w^{2}$ spatial nodes. The vector $\mathbf{f}_{t} \in \mathbb{R}^{2 n}$ denotes the components of the image mismatch gradient w.r.t. the displacement at time $t$. The elements of the vector $\mathbf{b}_{\lambda_{t}}$ depend on the boundary conditions and in our case $\mathbf{b}_{\lambda_{t}}=\mathbf{0}$.

The matrix $\mathbf{P}_{\lambda_{t}} \in \mathbb{R}^{2 n \times 2 n}$ can be expressed via finite-difference matrices $\mathbf{T}_{1}$ and $\mathbf{T}_{2}$ :

$$
\mathbf{P}_{\lambda_{t}}=\left(\begin{array}{cc}
\left(\lambda_{t}+2\right) \mathbf{T}_{1}^{T} \mathbf{T}_{1}+\mathbf{T}_{2}^{T} \mathbf{T}_{2} & \left(\lambda_{t}+1\right) \mathbf{T}_{1}^{T} \mathbf{T}_{2} \\
\left(\lambda_{t}+1\right) \mathbf{T}_{2}^{T} \mathbf{T}_{1} & \left(\lambda_{t}+2\right) \mathbf{T}_{2}^{T} \mathbf{T}_{2}+\mathbf{T}_{1}^{T} \mathbf{T}_{1}
\end{array}\right)
$$

The role of the matrix $\mathbf{T}_{1}$ can be interpreted as follows. Each displacement component can be considered as a separate image, whose columns could be joined into a single vector. Multiplying such a vector by the matrix $\mathbf{T}_{1}$ from the left produces a vector which contains the central-difference approximation of the derivatives of the displacement component at every internal node in the $x_{1}$-direction. The matrix $\mathbf{T}_{2}$ estimates the derivatives in the $x_{2}$-direction. Eq. (7) indicates that the matrix $\mathbf{P}_{\lambda_{t}}$ will be positive semi-definite at least when $\lambda_{t} \in$ $[-1, \infty)$.

Intuitively, we can view Eq. (6) as a filter of the noisy vector $\mathbf{f}_{t}$. The following probabilistic interpretation of Eq. (6) helps to develop criterion for the Lamé moduli in a systematic way:

$$
\begin{aligned}
p\left(\mathbf{f}_{t} \mid \boldsymbol{\Delta} \mathbf{u}_{t}, \sigma^{2}\right) & =\mathcal{N}\left(\boldsymbol{\Delta} \mathbf{u}_{t}, \sigma^{2} \mathbf{I}\right), \\
p\left(\boldsymbol{\Delta} \mathbf{u}_{t} \mid \mathbf{x}, \lambda_{t}, \sigma^{2}\right) & =\mathcal{N}\left(\mathbf{0}, \sigma^{2}\left(\mathbf{P}_{\lambda_{t}}-\mathbf{I}\right)^{-1}\right) .
\end{aligned}
$$

Eq. (8) assumes that the vector $\mathbf{f}_{t}$ is contaminated by additive Gaussian noise, whose variance is denoted by $\sigma^{2}$. Eq. (9) states that the displacement increments follow zero-mean Gaussian random process $\boldsymbol{\Delta} \mathbf{u}_{t}$ with the covariance matrix $\mathbf{K} \equiv \sigma^{2}\left(\mathbf{P}_{\lambda_{t}}-\mathbf{I}\right)^{-1} \in R^{2 n \times 2 n}$. Eq. (6) now can be re-obtained by applying conditioning [6] of the actual displacement increment $\boldsymbol{\Delta} \mathbf{u}_{t}$ on the observation vector $\mathbf{f}_{t}$ :

$$
\boldsymbol{\Delta} \mathbf{u}_{t}^{*} \equiv E\left[\boldsymbol{\Delta} \mathbf{u}_{t} \mid \mathbf{f}_{t}\right]=\mathbf{K}\left(\mathbf{K}+\sigma^{2} \mathbf{I}\right)^{-1} \mathbf{f}_{t}=\mathbf{P}_{\lambda_{t}}^{-1} \boldsymbol{\Delta} \mathbf{f}_{t} .
$$


Eq. (6) defines the mean of the displacement increment $\Delta \mathbf{u}_{t}$, whereas Eqs. (8) and (8) complete it to a full probability distribution. The kernel matrix $\mathbf{K}$ is not defined uniquely in $\mathbf{P}_{\lambda_{t}}$, but choosing $\sigma^{2}=\left\|\mathbf{f}_{t}-\boldsymbol{\Delta} \mathbf{u}_{t}^{*}\right\|^{2} /(2 n)$ removes the ambiguity.

This interpretation of fluid motion is useful because we can impose a noninformative improper hyper-prior on the Lamé ratio and maximize the loglikelihood (logevidence) $p\left(\mathbf{f}_{t} \mid \lambda\right)$ of the vector $\mathbf{f}_{t}$ w.r.t. the Lamé ratio $\lambda$. This procedure can be shown to be equivalent to the following minimization problem:

$$
\lambda_{t}^{*}=\arg \min _{\lambda}\{\underbrace{\frac{1}{2} \ln \frac{\left|\mathbf{P}_{\lambda}\right|}{\left|\mathbf{P}_{\lambda}-\mathbf{I}\right|}}_{\text {'Incompressibility' }}+\underbrace{n \ln 2 \pi \sigma^{2}}_{\text {'Deregularization' }}+\underbrace{\frac{1}{2 \sigma^{2}}\left(\boldsymbol{\Delta} \mathbf{u}_{t}^{*}\right)^{T} \mathbf{P}_{\lambda} \overbrace{\left(\mathbf{f}_{t}-\boldsymbol{\Delta} \mathbf{u}_{t}^{*}\right)}^{\text {'Noise' }}}_{\text {'Decorrelation' }}\} .
$$

We emphasize that Eq. (6) defines the mean function, and thus there are infinitely many possibilities for its probabilistic interpretation. As an example, one could consider a degenerate variant of the GP model Eqs. (8) and (9) which would result in the mean function $\Delta \mathbf{u}_{t}^{*} \equiv E\left[\Delta \mathbf{u}_{t} \mid \mathbf{f}_{t}\right]$ equivalent to Eq. (6) in the same way as Eq. (10):

$$
\begin{aligned}
& p\left(\boldsymbol{\Delta} \mathbf{u}_{t} \mid \mathbf{x}, \lambda_{t}\right)=\mathcal{N}\left(\mathbf{0}, \mathbf{P}_{\lambda_{t}}^{-1}\right), \quad \mathbf{f}_{t}=\mathbf{P}_{\lambda_{t}} \boldsymbol{\Delta} \mathbf{u}_{t} \sim \mathcal{N}\left(\mathbf{0}, \mathbf{P}_{\lambda_{t}}\right), \\
& \lambda_{t}^{*}=\arg \min _{\lambda}\left\{\frac{1}{2} \ln \left|\mathbf{P}_{\lambda}\right|+\frac{1}{2} \mathbf{f}_{t}^{T} \boldsymbol{\Delta} \mathbf{u}_{t}^{*}+n \ln 2 \pi\right\} .
\end{aligned}
$$

The GP model in Eqs. (8) and (9) is more justified than its degenerate counterpart, clf. the argument presented in [8]. A more thorough analysis of Eq. (13) would show that it could be qualitatively similar to Eq. (11), but the utilization of Eq. (11) would require a proper scaling of the matrix $\mathbf{P}_{\lambda_{t}}$.

\subsection{Computational Analysis}

Let us analyze Eq. (7) and the above-derived Bayesian evidence criterion Eq. (11). Fig. 11indicates how the smallest eigenvalues of the matrices $\mathbf{P}_{\lambda}$ and $\mathbf{K}+\sigma^{2} \mathbf{I}=$ $\left(\mathbf{P}_{\lambda}-\mathbf{I}\right)^{-1} \mathbf{P}_{\lambda}$ depend on the Lamé ratio $\lambda$ and grid size. The eigenvalues are estimated on several small grids, where the interplay between the ellipticity and point-wise stability is the most notable. Fig. 1] shows that the range of allowed Lamé ratio values is confined by the point-wise stability of the Lamé functional stated in Eq. (5) to the interval $\lambda \in(-1.5, \infty)$. Notably, the ellipticity constraint $\lambda \in(-2, \infty)$ does not ensure the positive definiteness of the matrix $\mathbf{P}_{\lambda}$, yet the smallest singular value is never equal to zero and therefore a unique solution of Eq. (6) exists even when $\lambda \in(-2,-1.5]$. However, Fig. 1b indicates that the covariance matrix of the GP model in Eq. (9) is surely positive definite only when $\lambda \in(-1.5, \infty)$.

The estimates of the logdeterminants of the matrix $\mathbf{P}_{\lambda}$ and the covariance matrix of the GP model are presented in Figs. 1, d. In the case of a larger $126 \times 126$ grid, the values are estimated by first applying sparse LU decomposition and then summing all the logarithms of the diagonal elements in the U-matrix. 


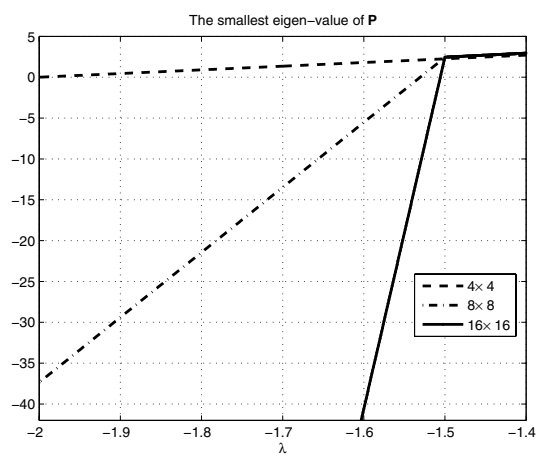

(a)

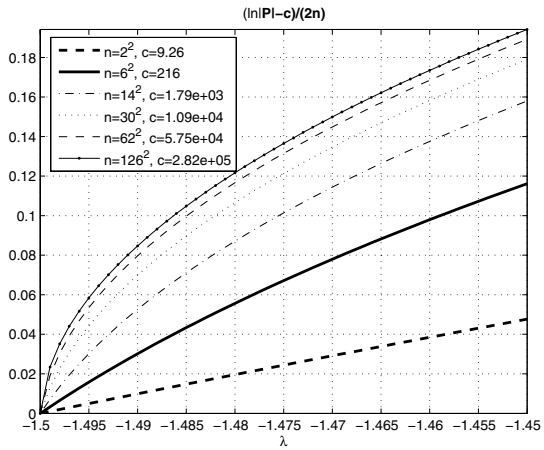

(c)

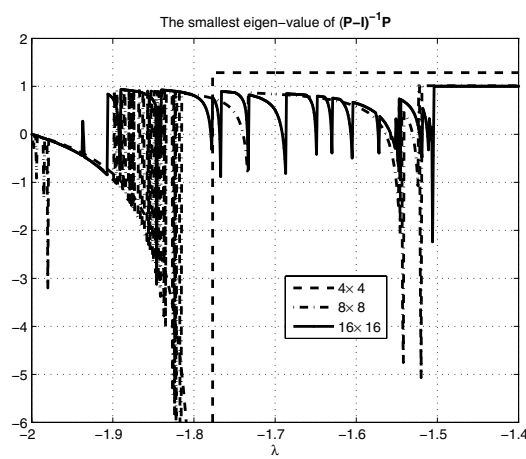

(b)

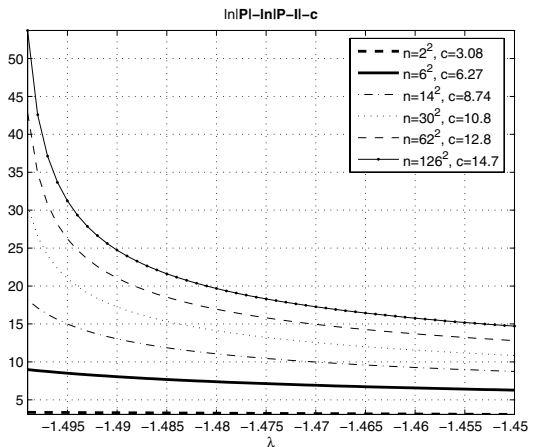

(d)

Fig. 1. (a) The dependence of the smallest eigenvalue of $\mathbf{P}_{\lambda}$ on the ratio of the Lamé moduli $\lambda$, and (b) similar analysis concerning the range of positive-definiteness of the covariance matrix of the GP model Eqs. (8) and (9). (c) The dependence of the logdeterminant $\ln \left|\mathbf{P}_{\lambda}\right|$ on the ratio of the Lamé moduli $\lambda$, (d) the difference between the log-determinants in Eq. (11)

In connection with Eq. (11), Fig. 11d indicates that a priori we should give preference to large values $\lambda$, i.e. the flow is supposed to be incompressible. However, at the same time we seek for the displacement increment $\boldsymbol{\Delta} \mathbf{u}_{t}$ most similar to the force field $\mathbf{f}_{t}$, which naturally would require smaller values of $\lambda$. The implications of this balance to an example problem will be analyzed in Section 4 .

\subsection{Extended Fluid Motion Estimation}

Below we will present a skeleton of the fluid motion estimation algorithm with adaptable Lamé moduli:

Initialize $\epsilon, \kappa$, set $t=0, \mathbf{u}_{0}=\mathbf{0}$.

WHILE $\left\|\varrho_{t}(\mathbf{x})-\varrho_{T}(\mathbf{x})\right\|_{2}>\epsilon$,

Compute the vector $\mathbf{f}_{t}$, 


$$
\begin{aligned}
& \left(\boldsymbol{\Delta} \mathbf{u}_{t}, \lambda_{t}, \sigma_{t}^{2}\right)=\arg \min _{\boldsymbol{\Delta} \mathbf{u}, \lambda, \sigma^{2}}\left\{\frac{1}{2} \ln \frac{\left|\mathbf{P}_{\lambda}\right|}{\left|\mathbf{P}_{\lambda}-\mathbf{I}\right|}+n \ln 2 \pi \sigma^{2}+\frac{1}{2 \sigma^{2}}\left(\Delta \mathbf{u}_{t}^{*}\right)^{T} \mathbf{P}_{\lambda}\left(\mathbf{f}_{t}-\boldsymbol{\Delta} \mathbf{u}_{t}^{*}\right)\right\}, \\
& \text { subject to }\left\{\begin{array}{l}
\mathbf{P}_{\lambda} \boldsymbol{\Delta} \mathbf{u}=\mathbf{f}_{t},\left.\quad \boldsymbol{\Delta} \mathbf{u}\right|_{\partial \Omega}=\mathbf{0}, \\
\|\boldsymbol{\Delta} \mathbf{u}\|_{2} \leq \kappa, \lambda>-1.5, \\
\sigma^{2}=\left\|\mathbf{f}_{t}^{T}-\boldsymbol{\Delta} \mathbf{u}\right\|^{2} /(2 n) .
\end{array}\right. \\
& \mathbf{u}_{t}=\mathbf{u}_{t}+\boldsymbol{\Delta} \mathbf{u}_{t}, \\
& t \leftarrow t+d t, \\
& \text { END. }
\end{aligned}
$$

This optimization problem is hard to solve due to the large number of equality constraints and the strong nonlinear coupling of the Lamé moduli with the displacement increments. For example, Section 4 states a problem that requires $2 n=2 \cdot 400^{2}$ equality constraints which is far beyond the limits of any generalpurpose constrained optimization routine.

To circumvent these difficulties, we perform the minimization in Eq. (11) by direct evaluation of the logevidence over a small number of characteristic values of the Lamé moduli that we believe sufficiently cover the optimization region $\lambda \in[-2, \infty]$. We note that the point $\lambda=-1$ splits the ellipticity region of the Lamé functional into sub-elliptic and super-elliptic smoothing. Thus, we first solve $\mathbf{P}_{\lambda} \boldsymbol{\Delta} \mathbf{u}=\mathbf{f}_{t}$ for a certain $\boldsymbol{\Delta} \mathbf{u}_{t}$ dependent on $\lambda$ by applying pseudoinverse techniques based on the sparse LU decomposition, and later set $\sigma_{t}^{2}=$ $\left\|\mathbf{f}_{t}-\boldsymbol{\Delta} \mathbf{u}\right\|^{2} /(2 n)$. The logevidence is then evaluated and the computation is repeated for different values of the Lamé ratio. Finally the best displacement increment and the optimal ratio $\lambda_{t}$ are chosen which minimize the criterion in Eq. (11).

\section{Experiments}

\subsection{Circle to Letter 'c' Matching}

Finding the deformation field between a circle and a letter ' $c$ ' is a synthetic problem frequently used to assess the quality of various image registration algorithms 22. The template circle $\varrho_{0}(\mathbf{x})$ and target 'c' $\varrho_{T}(\mathbf{x})$ images were generated as described in [2]. The fluid registration algorithm has been applied three times, each time with fixed Lamé parameter values arising from one of the three zones of the ellipticity condition: (i) nearly incompressible flow, (ii) Laplacian smoothing, and (iii) weakly-elliptic flow. The results presented in Fig. 2 show that the displacements strongly depend on the Lamé moduli. Fig. 21 depicts the flow of the nearly incompressible fluid flow. The divergence of displacement increments is penalized by a large $\lambda$ value, and the flow generates two vortices which can be seen in the left part of the letter 'c' image. The relatively smoothest transformation is achieved in the Laplacian case, shown in Fig. 2b, whereas sub-elliptic flow results in source points shown in Fig. 2r. It is important to note that all of the final displacement maps carry the circle into 'c' perfectly, while being very different in their nature, depending on what prior knowledge we assume about the flow. 

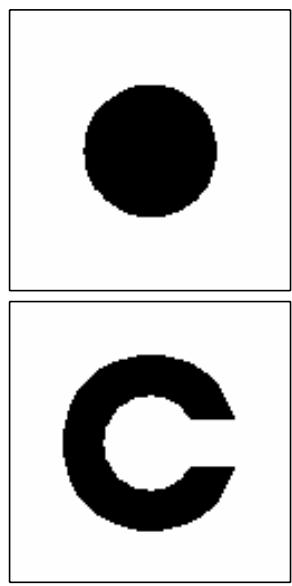

(a)

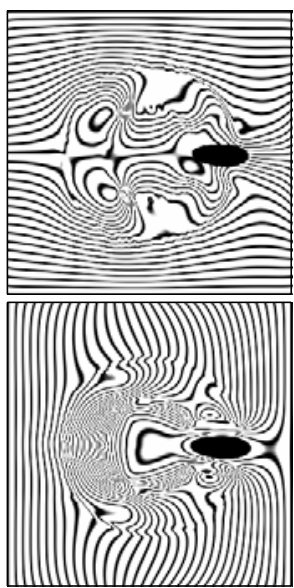

(b)

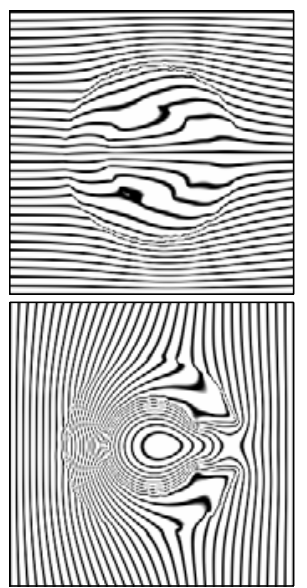

(c)

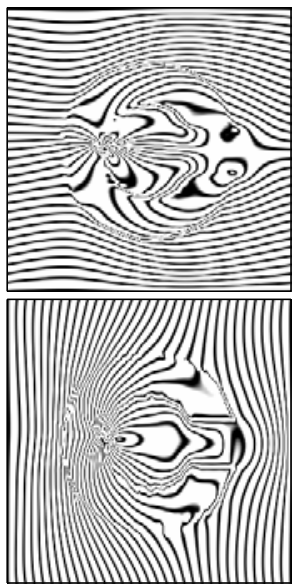

(d)

Fig. 2. Diversity of fluid motions. (a) Circle to 'c' matching results with: (b) $\lambda=10$ strong ellipticity (nearly incompressible) fluid flow, (c) $\lambda=-1$ Laplacian smoothing, (d) $\lambda=-1.9$ sub-elliptic flow. (b)-(d) The columns from left to right depict deformations of horizontal and vertical grids. All maps deform circle into letter 'c' perfectly

The experiments indicate that the use of the Lamé functional $\mathcal{R}_{\mu, \lambda}$ does not ensure diffeomorphic transformations in general. In fact, all maps develop singularities at relatively early stages of image matching. Symmetry breaking is also notable in all the three cases. The divergence of the displacement increments is often advocated as a term responsible for the image intensity appearance/disappearance rate. If one superimposed the deformed circles in all three cases after few iterations, one could see that the circle expands to the borders of letter ' $c$ ' faster in the cases of the Laplacian and weak ellipticity models than in the case of the hyper-elliptic model. However, such a fact is of minor importance compared to the solenoidal nature of the flow imposed by large $\lambda$ values.

\subsection{Interpolation of Motion in Jupiter Storms}

Interpolation of time events occurring in the atmosphere of astronomic bodies is a very potential application area of fluid motion estimation algorithms. Below we present the problem of interpolating the cloud motion around the Great Red Spot of the planet Jupiter. The first one of seven frames taken by the Cassini-Huygens spacecraft in one rotation period of the planet of Jupiter is shown in Fig. 4.2 a. We assume that the exact time of photographing of the images is not known. For simplicity, we estimate the optical flow from the frame No. 1 to the frame No. 7, and try to re-produce the frames No. 2-6 by evaluating deformations which are made equidistant in space. The purpose 


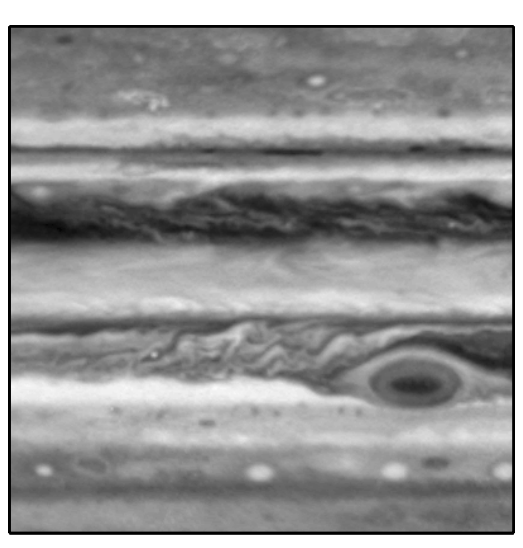

(a)

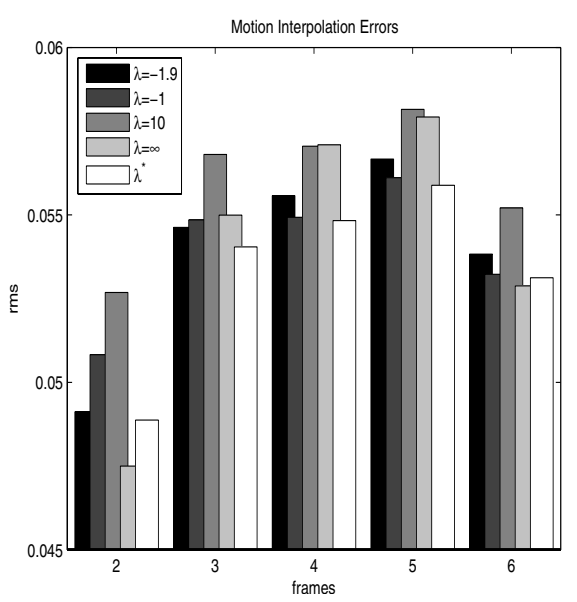

(b)

Fig. 3. An image of Jupiter storms near the Great Red Spot taken by Cassini-Huygens spacecraft in November 2000. Each image is of a resolution $400 \times 400$ pixels and span 100 degrees around the equator both in East-West and North-South directions [1. (a) the first frame and (b) the root-mean-square errors between the five estimated and real frames No.2-6

of this example is to show that the optimization of the Bayesian evidence criterion reduces the model errors even when our model a priori makes the strong, possibly invalid, assumption on the constant brightness of the Jupiter storm motion.

The resulting root-mean-squared errors are indicated in Fig. 4.2 b. They show that at different time instances different parameter settings are optimal. In the beginning, the motion appears to be incompressible, but later divergent flow produces smaller errors in the frames No. 3-5. Also, there is a notable difference between the results obtained with the ratio $\lambda=-1$ and the divergence-free flow of $\lambda=\infty$, the latter simulated by using the classical projection of the displacement increment onto the divergence-free subspace. The Laplacian flow $\lambda=-1$ is inferior to our approach in all frames.

It is notable that the optimal temporal reshaping of the Lamé moduli, denoted by $\lambda^{*}$, produces better image interpolation results than any one of the fixed value cases. However, the difference is rather small for several reasons. First, the constant image brightness assumption in Eq. (1) is hardly valid, at least because of the bright dots appearing in the left of the Great Red Spot which are believed to be lightnings [1. Second, it is clear that the flow around the Great Red Spot is very rotational whereas in other areas it is divergent or simply affine. The problem of the interpolation of Jupiter cloud motion would therefore require spatial models for the Lamé moduli. The task of associating different areas with different flow regimes corresponding to particular Lamé moduli will for now remain as an open question. 


\section{Conclusions}

This work has focused on the analysis of fluid-based estimation of motion in images, put into a probabilistic form. The negative spatial gradient of the image mismatch has been interpreted as a time-dependent Gaussian process whose temporal covariance structure depends on the second variation of the Lamé functional. Under this model, the ratio of the Lamé moduli has become a hyperparameter that maximizes the Bayesian evidence of the postulated temporal GP model. More precisely, in the absence of any specific knowledge, the Lamé ratio can be chosen to minimize at each time instant the difference between the fluid flow increment and the external force field, i.e. the negative image gradient. At the same time, the flow should be kept almost incompressible.

The above-discussed optimal reshaping of the Lamé moduli is only an approximative Bayesian inference procedure, and its practical value to the determination of the optimal fluid flow regime remains unclear. The presented method indicates how one can determine the amount of temporal compressibility of the fluid flow by decorrelating the displacement increment with the noise term, which is the difference between the external force field and the estimated displacement increment. When performing the experiments with Jupiter storm images, we have observed that the Lamé ratio introduces a trade-off between the abovementioned correlation and incompressibility. However, the evidence values are rather flat on the whole region of Lamé values, and the improvements are small.

There are two interesting consequences of this study. First, a priori fixing the compressibility of the imaged motion is always suboptimal. Neither typical incompressible flow $\lambda / \mu=\infty$, nor the 'most assumptionless' $\lambda / \mu=-1$ Laplacian flow could produce best results. Second, the flatness of the Bayesian evidence criterion can be utilized to detect inadequacies in the model of the image intensity evolution, in particular, validating the optical flow constraint.

We believe that the optimization of the Lamé moduli is important whenever: (i) the postulated model of the image mismatch measure strongly deviates from its true unknown counterpart or (ii) the spatial dependence of the Lamé moduli cannot be neglected but is hard to identify.

\section{References}

1. The Great Red Spot movie. NASA's Planetary PhotoJournal, PIA02829, NASA/JPL/University of Arizona, November 2002.

2. G. Christensen. Deformable shape models for anatomy. Phd thesis, Washington University, 1994.

3. T. Corpetti, É. Mémin, and P. Pérez. Dense estimation of fluid flows. IEEE Trans. on Pattern Analysis and Machine Intelligence, 24(3):365-380, 2002.

4. G.S. Cunningham, A. Lehovich, and K.M. Hanson. Bayesian estimation of regularization parameters for deformable surface models. SPIE, pages 562-573, 1999.

5. B. D.Reddy. Introductory functional analysis : with applications to boundary value problems and finite elements. Springer, 1998. 
6. M.N. Gibbs. Bayesian Gaussian Processes for Regression and Classification. Ph.d. thesis, Cambridge University, 1997.

7. A. Hagemann. A Biomechanical Model of the Human Head with Variable Material Properties for Intraoperative Image Correction. Logos Verlag Berlin, 2001.

8. J. Quiñonero-Candela and C. E. Rasmussen. Analysis of some methods for reduced rank gaussian process regression. In R. Shorten and R. Murray-Smith, editors, Proc. of Hamilton Summer School on Switching and Learning in Feedback systems. Springer-Verlag, 2004.

9. Pengcheng Shi and Huafeng Liu. Stochastic finite element framework for simultaneous estimation of cardiac kinematic functions and material parameters. Medical Image Analysis, 7:445-464, 2003. 